TRANSACTIONS OF THE

AMERICAN MATHEMATICAL SOCIETY

Volume 359, Number 7, July 2007, Pages 3251-3273

S 0002-9947(07)04137-2

Article electronically published on February 13, 2007

\title{
THE MCMULLEN DOMAIN: RINGS AROUND THE BOUNDARY
}

\author{
ROBERT L. DEVANEY AND SEBASTIAN M. MAROTTA
}

\begin{abstract}
In this paper we show that there are infinitely many rings $\mathcal{S}^{k}, k \geq$ 1 , around the McMullen domain in the parameter plane for the family of complex rational maps of the form $z^{n}+\lambda / z^{n}$ where $\lambda \in \mathbb{C}$ and $n \geq 3$. These rings converge to the boundary of the McMullen domain as $k \rightarrow \infty$. The rings $\mathcal{S}^{k}$ contain $(n-2) n^{k-1}+1$ parameter values that lie at the center of Sierpinski holes. That is, these parameters lie at the center of an open set in the parameter plane in which all of the corresponding maps have Julia sets that are Sierpinski curves. The rings also contain the same number of superstable parameter values, i.e., parameter values for which one of the critical points is periodic of period either $k$ or $2 k$.
\end{abstract}

Our goal in this paper is to consider the dynamics of families of rational maps of the form

$$
F_{\lambda}(z)=z^{n}+\frac{\lambda}{z^{n}}
$$

where $\lambda \neq 0$ is a complex parameter and $n$ is a positive integer. The Julia sets corresponding to maps in these families have been shown to possess a number of interesting dynamical and topological properties.

In this paper we discuss some of the properties of the parameter plane for these maps. Each of these maps has $2 n$ "free" critical points. However, like the wellstudied quadratic family $Q_{c}(z)=z^{2}+c$, each of these families has only one free critical orbit since all forward orbits of the critical points behave symmetrically. Hence the $\lambda$-plane is a natural parameter plane for these families.

As another similarity with the quadratic family, the point at $\infty$ is a superattracting fixed point for each $\lambda$ when $n>1$, and so it may be the case that the critical orbits enter the basin of this fixed point. Unlike the quadratic family, there are three distinctly different manners in which the critical orbit may escape to $\infty$, and this in turn determines the topological structure of the Julia sets for the escape parameters. We denote the immediate basin of attraction of $\infty$ by $B_{\lambda}$. One possible escape scenario is that the critical values all lie in $B_{\lambda}$. Since 0 is a pole, there is a neighborhood of 0 that is mapped into $B_{\lambda}$. Now either this neighborhood is itself contained in $B_{\lambda}$, or else 0 lies in a disjoint preimage of $B_{\lambda}$ which we denote by $T_{\lambda}$. In the latter case, we note that $F_{\lambda}$ maps $T_{\lambda}$ in an $n$-to-one fashion onto $B_{\lambda}$ while $F_{\lambda} \mid B_{\lambda}$ is also $n$-to-one. Hence the only preimages of $B_{\lambda}$ are $B_{\lambda}$ itself and $T_{\lambda}$. A second possible scenario occurs when the critical values lie in $T_{\lambda}$, and a third possibility arises when some higher iterate of the critical values lies in $T_{\lambda}$. The following Theorem describes the Julia sets that result from these three different situations (see [6]).

Received by the editors May 5, 2005.

2000 Mathematics Subject Classification. Primary 37F10; Secondary 37F45.

(C)2007 American Mathematical Society Reverts to public domain 28 years from publication 3251 
Theorem (The Escape Trichotomy). For the family of functions

$$
F_{\lambda}(z)=z^{n}+\frac{\lambda}{z^{n}}
$$

with $n \geq 2$ and $\lambda \in \mathbb{C}$ :

1. If the critical values lie in $B_{\lambda}$, then the Julia set is a Cantor set.

2. If the critical values lie in $T_{\lambda} \neq B_{\lambda}$, then the Julia set is a Cantor set of simple closed curves.

3. If the critical values lie in any other preimage of $T_{\lambda}$, then the Julia set is a Sierpinski curve.

A Sierpinski curve is a planar set that is characterized by the following five properties: it is a compact, connected, locally connected and nowhere dense set whose complementary domains (of which there must be at least two) are bounded by simple closed curves that are pairwise disjoint. It is known from work of Whyburn [15] that any two Sierpinski curves are homeomorphic. In fact, they are homeomorphic to the well-known Sierpinski carpet fractal. From the point of view of topology, a Sierpinski curve is a universal set in the sense that it contains a homeomorphic copy of any planar, compact, connected, one-dimensional set. The first example of a Sierpinski curve Julia set was given by Milnor and Tan Lei 12 .

Case 2 of the Escape Trichotomy was first observed by McMullen [9, who showed that this phenomenon occurs in each family provided that $n \neq 1,2$ and $\lambda$ is sufficiently small.

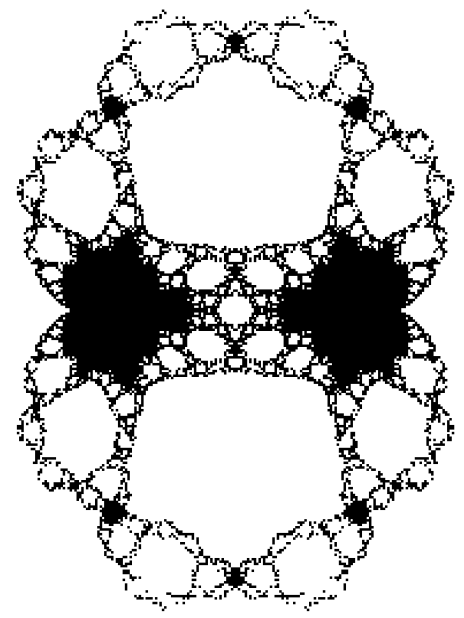

Figure 1 . The parameter plane for the family $z^{3}+\lambda / z^{3}$.

In Figure 1 we display the parameter plane for the family $F_{\lambda}(z)=z^{3}+\lambda / z^{3}$. The black regions indicate parameter values for which the critical orbit does not escape to $\infty$. Again in analogy with the quadratic polynomial family, for these parameters the Julia set is a connected set. The white regions in this picture represent $\lambda$ values for which the critical orbit tends to $\infty$. The exterior region corresponds to parameter values for which the Julia set is a Cantor set; we call this set the Cantor set locus. The small region in the center corresponds to parameter values for which the Julia set is a Cantor set of simple closed curves. We call this region 
the McMullen domain. The other white regions correspond to parameters for which the Julia set is a Sierpinski curve. These are called Sierpinski holes.

It is known that there are infinitely many disjoint Sierpinski holes for each of these families [1, [5] and that there is a parameter in each Sierpinski hole for which the orbit of the critical point lands on 0 at some iteration and therefore on $\infty$ at the next iteration, say at iteration $k>2$. We then call this $\lambda$-value the center of the Sierpinski hole and $k$ the escape time of the hole. All other parameters in a given Sierpinski hole have the property that the critical value has orbit that lands in $B_{\lambda}$ at the escape time iterate. By Whyburn's result, the Julia sets corresponding to any two parameters drawn from a Sierpinski hole are homeomorphic. However, as shown in [6], there exist Sierpinski holes corresponding to each escape time $k \geq 3$, and these have the property that if $\lambda_{1}$ and $\lambda_{2}$ lie in Sierpinski holes with different escape times, then $F_{\lambda_{1}}$ and $F_{\lambda_{2}}$ are not topologically conjugate on their Julia sets.

Note that, in the case $n=3$, there appear to be two large copies of a Mandelbrot set that straddle the positive and negative real axes. These are called the principal Mandelbrot sets for $F_{\lambda}$. Using the Douady-Hubbard theory of polynomial-like maps, it is known that these sets are actually homeomorphic to the standard quadratic Mandelbrot set 2 and that, for parameters drawn from these sets, there is an invariant subset on which $F_{\lambda}$ (or $F_{\lambda}^{2}$ ) is conjugate to the corresponding quadratic polynomial on its Julia set. Also apparent in this image are two large Sierpinski holes along the positive and negative imaginary axis. These holes have escape time 3.

Our goal in this paper is to investigate further properties of the parameter plane for these maps and, in particular, the structure of the parameter plane in a neighborhood of the McMullen domain. It is known [3] that there is a unique McMullen domain in the parameter plane for each $n \geq 3$, and this region is an open disk that is bounded by a simple closed curve.
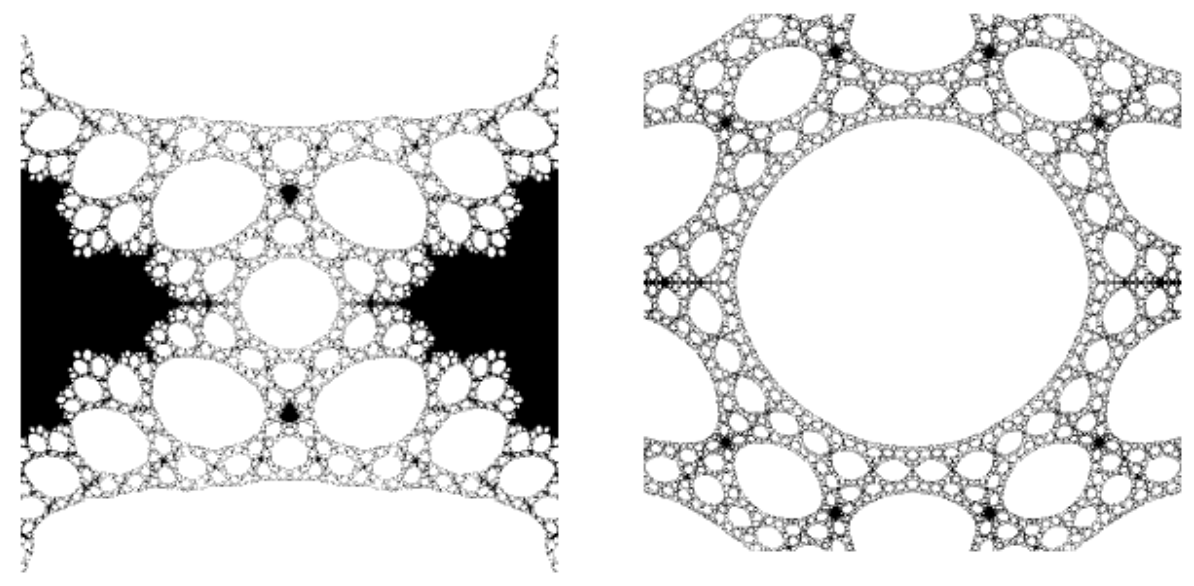

FIgURE 2. Magnifications of the parameter plane for the family $z^{3}+\lambda / z^{3}$ around the McMullen domain.

In Figure 2 we have displayed several magnifications of the region around the McMullen domain in the case $n=3$. In the first image, note that there are 
four large Sierpinski holes symmetrically placed around the McMullen domain. These Sierpinski holes all have escape time 4. Between the two upper and the two lower Sierpinski holes there appear to be small copies of a Mandelbrot set, while between the two left and two right holes we see the period two bulb of a principal Mandelbrot set and the remainder of the "tail" of this set. Indeed, one may draw a simple closed curve that encircles the McMullen domain and passes through the centers of each of these Sierpinski holes, the centers of the main cardioids of the two smaller Mandelbrot sets, and the centers of the two period two bulbs of the principal Mandelbrot sets. That is, on this simple closed curve, we find four parameter values for which $F_{\lambda}^{2}$ (or $F_{\lambda}^{4}$ ) has a superstable fixed point and four other values for which $F_{\lambda}^{4}$ maps the critical points to $\infty$, and these parameter values alternate between the superstable and the centers of Sierpinski holes as the parameter winds around the closed curve.

Inside these four Sierpinski holes appear to be another simple closed curve containing ten Sierpinski holes. Each of these holes has escape time 5. Also, each pair of these holes apparently has either a small copy of a Mandelbrot set or a portion of a principal Mandelbrot set between them. Examining the further magnification in Figure 2, we see a smaller closed curve containing 28 Sierpinski holes with escape time 6 and, inside that curve, an even smaller curve containing 82 Sierpinski holes with escape time 7 . It appears that the $k^{\text {th }}$ curve meets exactly $3^{k}+1$ Sierpinski holes with escape time $k+3$ as well as the same number of (portions of) Mandelbrot sets. We call these curves rings around the McMullen domain.
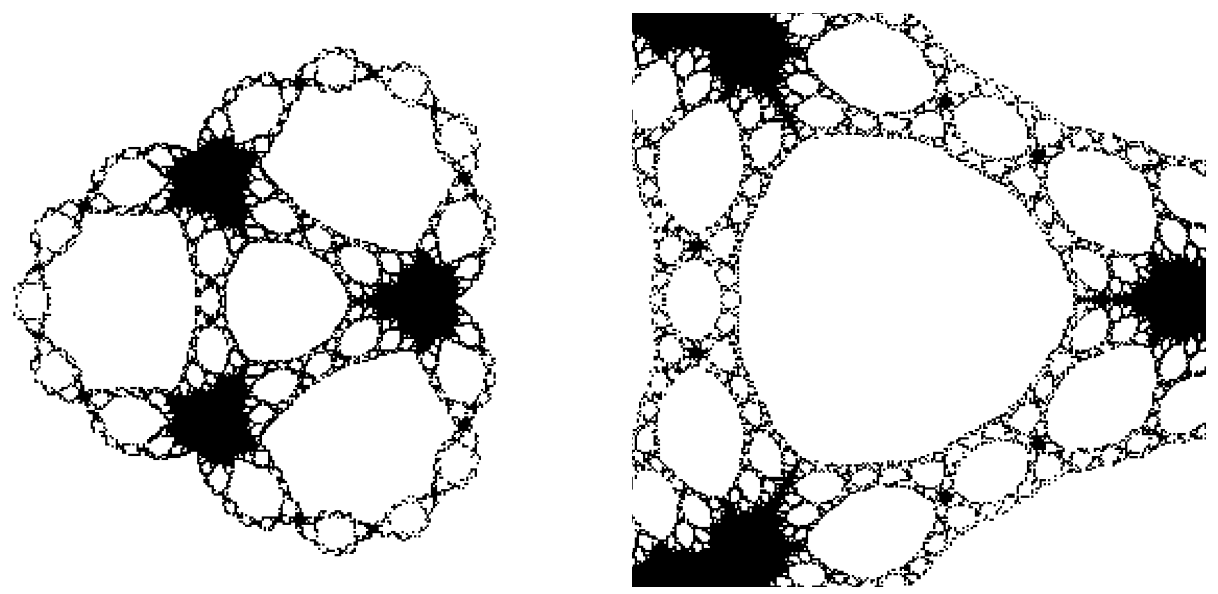

Figure 3. The parameter plane for the family $z^{4}+\lambda / z^{4}$ and a magnification around the McMullen domain.

Actually, the formula in the general case is a little more complicated than that. In Figure 3 we display the parameter plane for the case $n=4$ as well as a magnification of the McMullen domain. Here we see three principal Mandelbrot sets caught between three large Sierpinski holes, each of which has escape time 3. Inside these sets is a curve containing 9 Sierpinski holes, each with escape time 4, and inside another curve containing 33 holes of escape time 5 . Further magnification shows that there are $2 \cdot 4^{k-1}+1$ holes with escape time $k+2$ in the case $n=4$. 
Our main goal in this paper is to make these observations rigorous. We shall prove:

Theorem (Rings around the McMullen domain). For each $n \geq 3$, the McMullen domain for the family $z^{n}+\lambda / z^{n}$ is surrounded by infinitely many simple closed curves $\mathcal{S}^{k}$ for $k=1,2, \ldots$ having the property that:

1. Each curve $\mathcal{S}^{k}$ surrounds the McMullen domain as well as $\mathcal{S}^{k+1}$, and the $\mathcal{S}^{k}$ accumulate on the boundary of the McMullen domain as $k \rightarrow \infty$.

2. The curve $\mathcal{S}^{k}$ meets the centers of $\tau_{k}^{n}$ Sierpinski holes, each with escape time $k+2$, where

$$
\tau_{k}^{n}=(n-2) n^{k-1}+1 .
$$

3. The curve $\mathcal{S}^{k}$ also passes through $\tau_{k}^{n}$ superstable parameter values where a critical point is periodic of period $k$ or $2 k$.

In a subsequent paper [4] we shall show that these superstable parameter values each lie at the center of a small copy of a Mandelbrot set, while the Sierpinski holes surrounding the centers are all simply connected sets.

\section{Elementary mapping properties}

In this paper we restrict our attention to the family of rational maps given by

$$
F_{\lambda}(z)=z^{n}+\frac{\lambda}{z^{n}}
$$

where, in view of McMullen's result, we assume that $n \geq 3$.

In the dynamical plane, the object of principal interest is the Julia set of $F_{\lambda}$, which we denote by $J\left(F_{\lambda}\right)$. The Julia set is the set of points at which the family of iterates $\left\{F_{\lambda}^{n}\right\}$ fails to be a normal family in the sense of Montel. It is known that $J\left(F_{\lambda}\right)$ is also the closure of the set of repelling periodic points for $F_{\lambda}$ as well as the boundary of the set of points whose orbits escape to $\infty$ under iteration of $F_{\lambda}$. See 11.

The point at $\infty$ is a superattracting fixed point for $F_{\lambda}$, and we denote the immediate basin of $\infty$ by $B_{\lambda}$. It is well known that $F_{\lambda}$ is conjugate to $z \mapsto z^{n}$ in a neighborhood of $\infty$ in $B_{\lambda}$ [13. There is also a pole of order $n$ for $F_{\lambda}$ at the origin, so there is a neighborhood of 0 that is mapped into $B_{\lambda}$ by $F_{\lambda}$. If the full preimage of $B_{\lambda}$ that contains this neighborhood is disjoint from $B_{\lambda}$, then we denote the preimage of $B_{\lambda}$ that contains 0 by $T_{\lambda}$. So $F_{\lambda}$ maps both $B_{\lambda}$ and $T_{\lambda}$ in $n$-to-one fashion over $B_{\lambda}$. We call $T_{\lambda}$ the trap door, since any orbit that eventually enters the immediate basin of $\infty$ must "fall through" $T_{\lambda}$ enroute to $B_{\lambda}$.

Besides 0 and $\infty, F_{\lambda}$ has $2 n$ other critical points given by $\lambda^{1 / 2 n}$. We call these points the free critical points for $F_{\lambda}$. There are, however, only two critical values, and these are given by $\pm 2 \sqrt{\lambda}$. We denote a free critical point by $c_{\lambda}$ and a critical value by $v_{\lambda}$. The map also has $2 n$ prepoles given by $(-\lambda)^{1 / 2 n}$. Note that all of the critical points and prepoles lie on the circle of radius $|\lambda|^{1 / 2 n}$ centered at the origin. We call this circle the critical circle and denote it by $C_{\lambda}$.

The map $F_{\lambda}$ has some very special properties when restricted to circles centered at the origin. The following is a straightforward computation (see [3]). 
Proposition. $1 . F_{\lambda}$ takes the critical circle $2 n$-to-one onto the line interval connecting the two critical values $\pm \sqrt{2 \lambda}$;

2. $F_{\lambda}$ takes any other circle centered at the origin to an ellipse whose foci are the critical values.

We call the image of the critical circle the critical segment. We call the straight line connecting the origin to $\infty$ and passing through one of the critical points (resp., prepoles) a critical point ray (resp., prepole ray). Similar straightforward computations show that each of the critical point rays is mapped in two-to-one fashion onto one of the two straight line segments of the form $t v_{\lambda}$, where $t \geq 1$ and $v_{\lambda}$ is the image of the critical point on this ray. So the image of a critical point ray is a straight ray connecting either $v_{\lambda}$ or $-v_{\lambda}$ to $\infty$. Thus the critical segment together with these two rays forms a straight line through the origin.

Similarly, each of the $2 n$ prepole rays is mapped in one-to-one fashion onto the straight line given by $i t \sqrt{\lambda}$, where $t$ is now any real number. Note that the image of the prepole rays is the line that is perpendicular to the line $t v_{\lambda}$ for $t \in \mathbb{R}$, i.e., the line that contains the critical segment and the images of all of the critical point rays.

Let $U_{\lambda}$ be a sector bounded by two prepole rays corresponding to adjacent prepoles on $C_{\lambda}$, i.e., $U_{\lambda}$ is a sector in the plane with angle $2 \pi / 2 n$. We call $U_{\lambda}$ a critical point sector since it contains at its "center" a unique critical point of $F_{\lambda}$. Similarly, let $V_{\lambda}$ be the sector bounded by two critical point rays corresponding to adjacent critical points on $C_{\lambda}$. We call $V_{\lambda}$ a prepole sector. The next result follows immediately from the above.

Proposition (Mapping properties of $F_{\lambda}$ ). 1. $F_{\lambda}$ maps the interior of each critical point sector in two-to-one fashion onto the open half plane that is bounded by the image of the prepole rays and contains the critical value that is the image of the unique critical point in the sector;

2. $F_{\lambda}$ maps the interior of each prepole sector in one-to-one fashion onto the entire plane minus the two half lines $\pm t v_{\lambda}$ where $t \geq 1$;

3. $F_{\lambda}$ maps the region in either the interior or the exterior of the critical circle onto the complement of the critical segment as an $n$-to-one covering map of $\overline{\mathbb{C}}$ (except at 0 and $\infty$ ).

We now turn to the symmetry properties of $F_{\lambda}$ in both the dynamical and parameter planes. Let $\nu$ be the primitive $2 n^{\text {th }}$ root of unity given by $\exp (\pi i / n)$. Then, for each $j$, we have $F_{\lambda}\left(\nu^{j} z\right)=(-1)^{j} F_{\lambda}(z)$. Hence, if $n$ is even, we have $F_{\lambda}^{2}\left(\nu^{j} z\right)=F_{\lambda}^{2}(z)$ for each $j$. Therefore the points $z$ and $\nu^{j} z$ land on the same orbit after two iterations, and so their orbits have the same eventual behavior for each $j$. If $n$ is odd, the orbits of $F_{\lambda}(z)$ and $F_{\lambda}\left(\nu^{j} z\right)$ are either the same or else they are the negatives of each other after the first iteration. In either case it follows that the orbits of $\nu^{j} z$ behave symmetrically under $z \mapsto-z$ for each $j$. Hence the Julia set of $F_{\lambda}$ is always symmetric under $z \mapsto \nu z$. In particular, each of the free critical points eventually maps onto the same orbit (in the case $n$ is even) or onto one of two symmetric orbits (in the case $n$ is odd). Thus these orbits all have the same behavior, and so the $\lambda$-plane is a natural parameter plane for each of these families. Note also that, if $n$ is even and the orbit of some critical point eventually lands on some other critical point at iteration $j \geq 1$, then in fact one of the critical points of 
$F_{\lambda}$ must be periodic of period $j$. If $n$ is odd, then there are two possibilities: either one of the critical points has period $j$ or else it has period $2 j$.

Let $H_{\lambda}(z)$ be one of the $n$ involutions given by $H_{\lambda}(z)=\lambda^{1 / n} / z$. Then we have $F_{\lambda}\left(H_{\lambda}(z)\right)=F_{\lambda}(z)$, so that the Julia set is also preserved by each of these involutions. Note that each $H_{\lambda}$ maps the critical circle to itself and also fixes a pair of critical points of the form $\pm \sqrt{\lambda^{1 / n}}$. $H_{\lambda}$ also maps circles centered at the origin outside the critical circle to similar circles inside the critical circle and vice versa. It follows that two such circles, one inside and one outside the critical circle, are mapped onto the same ellipse by $F_{\lambda}$.

The parameter plane (see Figures 1 and 31) for $F_{\lambda}$ also possesses several symmetries. First of all, we have

$$
\overline{F_{\lambda}(z)}=F_{\bar{\lambda}}(\bar{z})
$$

so that $F_{\lambda}$ and $F_{\bar{\lambda}}$ are conjugate via the map $z \mapsto \bar{z}$. Therefore the parameter plane is symmetric under the map $\lambda \mapsto \bar{\lambda}$.

We also have $(n-1)$-fold symmetry in the parameter plane for $F_{\lambda}$. To see this, let $\omega$ be the primitive $(n-1)^{\mathrm{st}}$ root of unity given by $\exp (2 \pi i /(n-1))$. Then, if $n$ is even, we compute that

$$
F_{\lambda \omega}\left(\omega^{n / 2} z\right)=\omega^{n / 2}\left(F_{\lambda}(z)\right)
$$

As a consequence, for each $\lambda \in \mathbb{C}$, the maps $F_{\lambda}$ and $F_{\lambda \omega}$ are conjugate under the linear map $z \mapsto \omega^{n / 2} z$. In particular, since, when $\lambda$ is real, the real line is preserved by $F_{\lambda}$, it follows that the straight line passing through 0 and $\omega^{n / 2}$ is preserved by $F_{\lambda \omega}$.

When $n$ is odd, the situation is a little different. We now have

$$
F_{\lambda \omega}\left(\omega^{n / 2} z\right)=-\omega^{n / 2}\left(F_{\lambda}(z)\right) .
$$

Since $F_{\lambda}(-z)=-F_{\lambda}(z)$ when $n$ is odd, we therefore have that $F_{\lambda \omega}^{2}$ is conjugate to $F_{\lambda}^{2}$ via the map $z \mapsto \omega^{n / 2} z$. This means that the dynamics of $F_{\lambda}$ and $F_{\lambda \omega}$ are "essentially" the same, though subtly different. For example, if $F_{\lambda}$ has a fixed point, then under the conjugacy, this fixed point and its negative are mapped to a 2-cycle for $F_{\lambda \omega}$. Since the real line is invariant when $\lambda$ is real, it follows that the straight lines passing through the origin and $\pm \omega^{n / 2}$ are interchanged by $F_{\lambda \omega}$ and hence invariant under $F_{\lambda \omega}^{2}$.

To summarize the symmetry properties of $F_{\lambda}$, we have:

Proposition (Symmetries in the dynamical and parameter plane). The dynamical plane for $F_{\lambda}$ is symmetric under the map $z \mapsto \nu z$ where $\nu=\exp (\pi i / n)$. The parameter plane is symmetric under both $z \mapsto \bar{z}$ and $z \mapsto \omega z$ where $\omega=\exp (2 \pi i /(n-1))$.

The following result shows that the McMullen domain and all of the Sierpinski holes are located inside the unit circle in parameter space.

Proposition (Location of the Cantor set locus). Suppose $|\lambda| \geq 1$. Then $v_{\lambda}$ lies in $B_{\lambda}$ so that $\lambda$ lies in the Cantor set locus.

Proof. Suppose $|z| \geq 2|\lambda|^{1 / 2} \geq 2$. Then, since $|z| \geq|\lambda|^{1 / 2}$, we have

$$
\left|F_{\lambda}(z)\right| \geq|z|^{n}-\frac{|\lambda|}{|z|^{n}} \geq|z|^{n}-|\lambda|^{1-\frac{n}{2}} \geq|z|^{n}-1 \geq|z|^{n-1}>|z|
$$

since $n>2$. Hence $\left|F_{\lambda}^{j}(z)\right| \rightarrow \infty$, so the region $|z| \geq 2|\lambda|^{1 / 2}$ lies in $B_{\lambda}$. In particular, $v_{\lambda} \in B_{\lambda}$. 
For each $n$, let $\lambda^{*}=\lambda_{n}^{*}$ be the unique real solution to the equation

$$
\left|v_{\lambda}\right|=2|\sqrt{\lambda}|=|\lambda|^{1 / 2 n}=\left|c_{\lambda}\right| .
$$

Using this equation, we easily compute that

$$
\lambda^{*}=\left(\frac{1}{4}\right)^{\frac{n}{n-1}} .
$$

The circle of radius $\lambda^{*}$ plays an important role in the parameter plane, for if $\lambda$ lies on this circle, it follows that both of the critical values lie on the critical circle for $F_{\lambda}$. If $\lambda$ lies inside this circle, then $F_{\lambda}$ maps the critical circle strictly inside itself. We call the circle of radius $\lambda^{*}$ in parameter plane the dividing circle. We denote by $\mathcal{O}=\mathcal{O}_{n}$ the open set of parameters inside the dividing circle. We will be primarily concerned in later sections with values of the parameter that lie in $\mathcal{O}$. In particular, we shall show that all of the rings around the McMullen domain $\mathcal{S}^{k}$ with $k>1$ lie in this region while the ring $\mathcal{S}^{1}$ is the dividing circle itself.

\section{SOME SPECIAL CASES}

In this section we discuss the dynamics of several special cases of $F_{\lambda}$ that will help define the rings around the McMullen domain later.

First suppose that $\lambda$ lies on the dividing circle, i.e., $|\lambda|=\lambda^{*}$. In this case, all of the critical points, critical values, and prepoles of $F_{\lambda}$ lie on the same circle (the critical circle) in the dynamical plane, namely the circle

$$
|z|=\left(\frac{1}{2}\right)^{\frac{1}{n-1}}
$$

As $\lambda$ winds once around the dividing circle in the counterclockwise direction beginning on the real axis, the critical points and prepoles of $F_{\lambda}$ wind $1 / 2 n$ of a turn around the critical circle, while the critical values wind one-half of a turn around the critical circle, all in the counterclockwise direction. Hence there are exactly $n-1$ special parameter values on the dividing circle for which a critical point of the corresponding map equals a critical value, so for these special $\lambda$-values we have a superattracting fixed or period two point for $F_{\lambda}$. Equivalently, one computes that these $n-1$ parameters are given by

$$
\lambda=\left(\frac{1}{4}\right)^{\frac{n}{n-1}} .
$$

There are $n-1$ other parameters on this circle for which the critical value is a prepole, and these are given by

$$
\lambda=\left(\frac{-1}{4}\right)^{\frac{n}{n-1}} .
$$

This proves the case $k=1$ of the Rings Around the McMullen Domain Theorem.

Theorem. The ring $\mathcal{S}^{1}$ is the dividing circle in parameter plane. It contains $n-1$ superstable parameters and the same number of centers of Sierpinski holes.

See Figure 4

We next restrict our attention to values of $\lambda$ lying in $\mathbb{R}^{+}$. The graph of $F_{\lambda}$ shows that, in this case, $F_{\lambda}$ maps $\mathbb{R}^{+}$to itself and that there is a unique critical point lying in $\mathbb{R}^{+}$. We denote this critical point by $c_{0}=c_{0}(\lambda)$. See Figure 5 , 


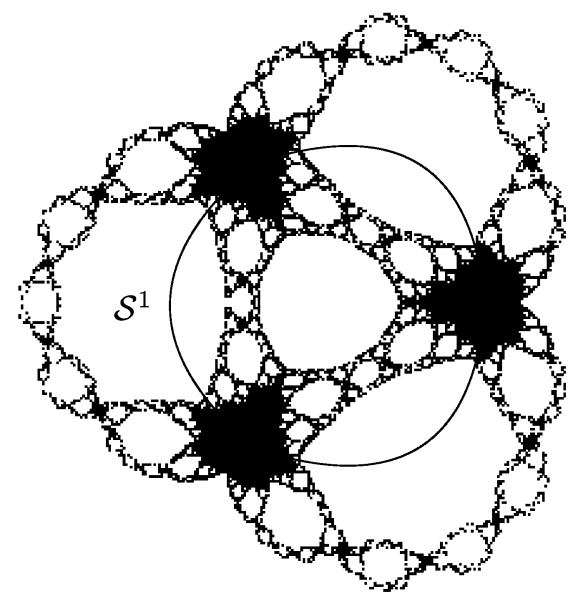

Figure 4 . The curve $\mathcal{S}^{1}$ in the parameter plane for $n=4$.

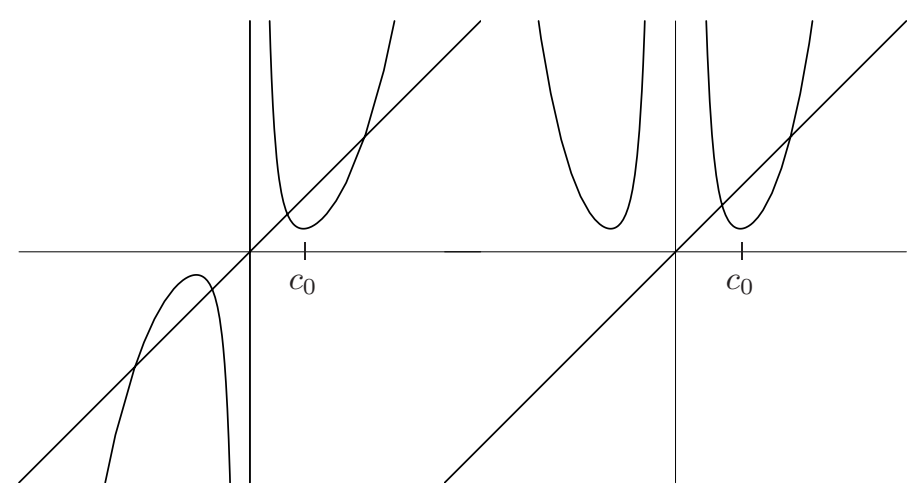

Figure 5. The graphs of $x^{3}+0.01 / x^{3}$ and $x^{4}+0.01 / x^{4}$.

It is known 22 that there is a Mandelbrot set whose central spine lies along the interval $\left[\lambda_{-}, \lambda_{+}\right]$contained in $\mathbb{R}^{+}$. Moreover, if $\lambda>\lambda_{+}$, then $\lambda$ lies in the Cantor set locus, whereas if $0<\lambda<\lambda_{-}$, then $\lambda$ lies in the McMullen domain. The graph of $F_{\lambda} \mid \mathbb{R}^{+}$shows that $F_{\lambda}$ undergoes a saddle-node bifurcation at $\lambda_{+}$and that the critical point $c_{\lambda}$ maps onto the repelling fixed point in $\partial B_{\lambda} \cap \mathbb{R}^{+}$after two iterations when $\lambda=\lambda_{-}$. Since each $F_{\lambda}$ is conjugate on the real line to a real quadratic polynomial of the form $Q_{c}(x)=x^{2}+c$, standard facts from quadratic dynamics yield the following.

Proposition (Superstable parameters for $\lambda \in \mathbb{R}^{+}$). There is a decreasing sequence of parameters $\lambda_{1}>\lambda_{2}>\ldots$ in $\mathbb{R}^{+}$converging to $\lambda_{-}$such that, for $\lambda=\lambda_{k}$, the critical point $c_{0}$ is periodic with period $k$ and the critical orbit in $\mathbb{R}^{+}$has the special form when $k \geq 2$ :

$$
0<v_{\lambda}=F_{\lambda}\left(c_{0}\right)<c_{0}=F_{\lambda}^{k}\left(c_{0}\right)<F_{\lambda}^{k-1}\left(c_{0}\right)<\ldots<F_{\lambda}^{3}\left(c_{0}\right)<F_{\lambda}^{2}\left(c_{0}\right) .
$$

In particular, $\lambda_{k}$ is a superstable parameter value of period $k$, and the orbit of $F_{\lambda_{k}}^{2}\left(c_{0}\right)$ is monotonically decreasing for $k-1$ iterations along $\mathbb{R}^{+}$. 
Portions of the graphs of $F_{\lambda_{k}}$ for $k=4$ and $k=8$ when $n=4$ are displayed in Figure 6. Note that the parameter $\lambda_{1}$ necessarily lies on the dividing circle $\mathcal{S}^{1}$. We shall show below that each $\lambda_{k}$ lies on $\mathcal{S}^{k}$.
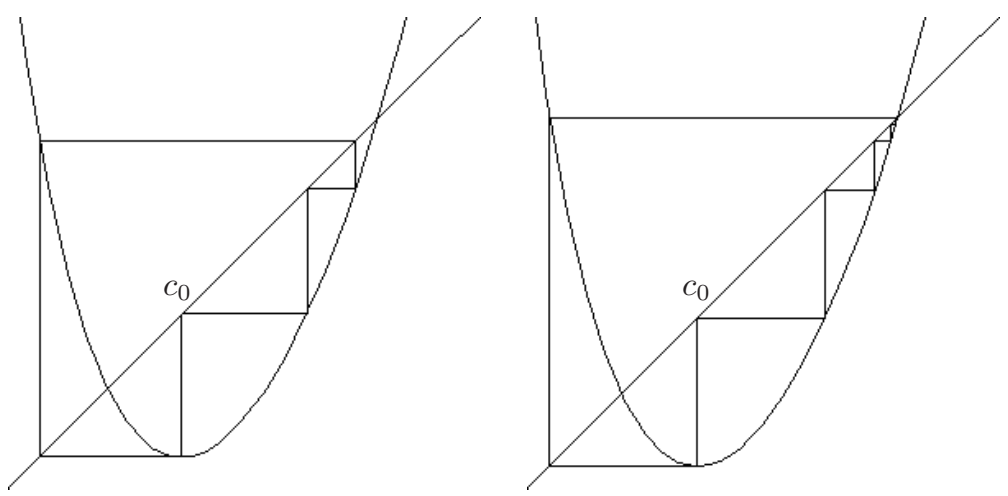

Figure 6 . The graphs of $F_{\lambda}$ for $\lambda=\lambda_{4}$ and $\lambda=\lambda_{8}$ when $n=4$.

Because of the $(n-1)$-fold symmetry in the parameter plane, we have a similar sequence of superstable parameter values along the ray $\lambda=\omega \cdot \mathbb{R}^{+}$in the parameter plane. To be more precise, first suppose that $n$ is even. Suppose that $\lambda=a \omega$ with $a>0$ and, as before, $\omega=\exp (2 \pi i /(n-1))$. Then, using the results in Section 1, we have that, if $t>0$,

$$
F_{\lambda}\left(\omega^{\frac{n}{2}} t\right)=\omega^{\frac{n}{2}} F_{a}(t)
$$

so that $F_{\lambda}$ on the line $\omega^{n / 2} \cdot \mathbb{R}^{+}$is conjugate to $F_{a}$ on $\mathbb{R}^{+}$.

Now $F_{\lambda}$ has critical points at

$$
\begin{aligned}
c_{0} & =(a \omega)^{\frac{1}{2 n}}, \\
c_{1} & =\nu(a \omega)^{\frac{1}{2 n}}, \\
c_{n+1} & =\nu^{n+1}(a \omega)^{\frac{1}{2 n}}=-\nu(a \omega)^{\frac{1}{2 n}}=-c_{1} .
\end{aligned}
$$

Note that the critical point $c_{n+1}$ lies on the line $\omega^{n / 2} \cdot \mathbb{R}^{+}$. This follows since

$$
\begin{aligned}
-\nu(a \omega)^{\frac{1}{2 n}} & =-(a)^{\frac{1}{2 n}}\left(\exp \left(\frac{\pi i}{n}\right) \exp \left(\frac{\pi i}{n(n-1)}\right)\right) \\
& =-(a)^{\frac{1}{2 n}} \exp \left(\frac{\pi i}{n-1}\right) \\
& =-a^{\frac{1}{2 n}} \omega^{\frac{1}{2}}=a^{\frac{1}{2 n}} \omega^{\frac{n}{2}} .
\end{aligned}
$$

Therefore the above Proposition goes over to the case where $\lambda=a \omega$ with $a=\lambda_{k} \in$ $\mathbb{R}^{+}$provided we now use the critical point $c_{n+1}$ lying on the line $\omega^{n / 2} \cdot \mathbb{R}^{+}$. We note that the symmetric critical point $c_{1}$ lies on the line $\omega^{1 / 2} \cdot \mathbb{R}^{+}$and maps onto the critical value on the line $\omega^{n / 2} \cdot \mathbb{R}^{+}$after one iteration.

The case where $n$ is odd is similar modulo the $z \mapsto-z$ symmetry described earlier. The difference is that the superattracting cycles now have period $2 k$ and alternate back and forth between $\omega \cdot \mathbb{R}^{+}$and $-\omega \cdot \mathbb{R}^{+}$. We have:

Proposition (Superstable parameters for $\lambda \in \omega \cdot \mathbb{R}^{+}$). Let $\lambda_{1}>\lambda_{2}>\ldots$ be the decreasing sequence in $\mathbb{R}^{+}$in the previous Proposition. Suppose $n$ is even. For 
$\lambda=\lambda_{k} \omega$, the critical point $c_{n+1}$ is periodic with period $k$ and the critical orbit along the line $\omega^{n / 2} \cdot \mathbb{R}^{+}$has the following special form when $k \geq 2$ :

$$
F_{\lambda}\left(c_{n+1}\right)<c_{n+1}=F_{\lambda}^{k}\left(c_{n+1}\right)<F_{\lambda}^{k-1}\left(c_{n+1}\right)<\ldots<F_{\lambda}^{3}\left(c_{n+1}\right)<F_{\lambda}^{2}\left(c_{n+1}\right) .
$$

In particular, $\lambda=\lambda_{k} \omega$ is a superstable parameter value of period $k$, and the orbit of $F_{\lambda}^{2}\left(c_{n+1}\right)$ is monotonically decreasing for $k-1$ iterations along $\omega^{n / 2} \cdot \mathbb{R}^{+}$. When $n$ is odd, replace $F_{\lambda}$ with $F_{\lambda}^{2}$. The cycle corresponding to $\lambda=\lambda_{k} \omega$ now has period $2 k$.

\section{Rings in the Dynamical Plane}

In this section we prove the existence of infinitely many rings $\gamma_{\lambda}^{k}$ for $k=0,1, \ldots$ in the dynamical plane. Each ring $\gamma_{\lambda}^{k}$ is a smooth, simple closed curve that is mapped $n^{k}$-to-one onto the critical circle by $F_{\lambda}^{k}$. We shall use these rings in the next section to construct the rings $\mathcal{S}^{k}$ in the parameter plane.

We begin by defining $\gamma_{\lambda}^{0}$ to be the critical circle. Recall that, if $\lambda \in \mathcal{O}$, then $F_{\lambda}$ maps $\gamma_{\lambda}^{0}$ strictly inside itself. Since all of the critical points of $F_{\lambda}$ lie on $\gamma_{\lambda}^{0}$, it follows that $F_{\lambda}$ takes the exterior of $\gamma_{\lambda}^{0}$ as an $n$-to-one covering onto the plane minus the critical segment and hence over the entire exterior of $\gamma_{\lambda}^{0}$. Thus there is a preimage $\gamma_{\lambda}^{1}$ lying outside of $\gamma_{\lambda}^{0}$ and mapped $n$-to-one onto $\gamma_{\lambda}^{0}$ by $F_{\lambda}$. Since $F_{\lambda}$ is a covering map, it follows that $\gamma_{\lambda}^{1}$ must be a single simple closed curve. Then $F_{\lambda}$ maps the exterior of $\gamma_{\lambda}^{1}$ as an $n$-to-one covering onto the exterior of $\gamma_{\lambda}^{0}$, so there is a preimage of $\gamma_{\lambda}^{1}$ lying in this region and mapped $n$-to-one to $\gamma_{\lambda}^{1}$. Call this simple closed curve $\gamma_{\lambda}^{2}$. Continuing inductively, we find a collection of simple closed curves $\gamma_{\lambda}^{k}$ for $k \geq 1$ having the properties that:

1. $\gamma_{\lambda}^{k+1}$ lies in the exterior of $\gamma_{\lambda}^{k}$;

2. $F_{\lambda}$ takes $\gamma_{\lambda}^{k+1}$ as an $n$-to-one covering onto $\gamma_{\lambda}^{k}$;

3. so $F_{\lambda}$ takes $\gamma_{\lambda}^{k+1}$ as an $n^{k+1}$-to-one covering of the critical circle;

4. the $\gamma_{\lambda}^{k+1}$ converge outward to the boundary of $B_{\lambda}$ as $k \rightarrow \infty$.

We now construct a parameterization of each of the $\gamma_{\lambda}^{k}$. In order for this parametrization to be well defined, we need to restrict our attention to parameters in the region $\mathcal{O}^{\prime}=\mathcal{O}-\left(-\lambda^{*}, 0\right]$, so that $-\pi<\operatorname{Arg} \lambda<\pi$. We therefore assume that $\lambda$ lies in $\mathcal{O}^{\prime}$ for the remainder of this paper.

For $\lambda \in \mathcal{O}^{\prime}$, there is a unique critical point of $F_{\lambda}$ lying in the region $|\operatorname{Arg} z|<$ $\pi / 2 n$. Call this critical point $c_{0}=c_{0}(\lambda)$. Note that $c_{0} \in \mathbb{R}^{+}$if $\lambda \in \mathbb{R}^{+}$. We index the remaining critical points by $c_{j}$ with the argument of $c_{j}$ increasing as $j$ increases.

To parametrize the critical circle $\gamma_{\lambda}^{0}$, we set $\gamma_{\lambda}^{0}(0)=c_{0}(\lambda)$. By the mapping properties Proposition, for each $\theta \in \mathbb{R}$, we then let $\gamma_{\lambda}^{0}(\theta)$ be the natural continuation of this parametrization of the circle in the counterclockwise direction. So $\gamma_{\lambda}^{0}(\theta)$ is $2 \pi$-periodic in $\theta$ and depends analytically on $\lambda$ for $\lambda \in \mathcal{O}^{\prime}$.

To parametrize $\gamma_{\lambda}^{1}(\theta)$, consider the portion of the critical point sector containing $c_{0}(\lambda)$ that lies outside the critical circle. There is a unique point in this region mapped to $c_{0}$ by $F_{\lambda}$; call this point $\gamma_{\lambda}^{1}(0)$. Then define $\gamma_{\lambda}^{1}(\theta)$ by requiring that

$$
F_{\lambda}\left(\gamma_{\lambda}^{1}(\theta)\right)=\gamma_{\lambda}^{0}(\theta)
$$

and that $\gamma_{\lambda}^{1}(\theta)$ varies continuously with $\theta$. Note that $\gamma_{\lambda}^{1}(\theta)$ is $2 n \pi$ periodic since $F_{\lambda}$ is $n$-to-one on $\gamma_{\lambda}^{1}$. We then proceed inductively to define $\gamma_{\lambda}^{k}(\theta)$ by first specifying that, in the outside portion of the critical point sector containing $c_{0}, \gamma_{\lambda}^{k}(0)$ is the unique point that is mapped by $F_{\lambda}$ to $\gamma_{\lambda}^{k-1}(0)$ and by then using $F_{\lambda}$ to complete 
this parameterization. As above, for each $k, \gamma_{\lambda}^{k}(\theta)$ is $2 n^{k} \pi$ periodic in $\theta$ and depends analytically on $\lambda$.

To prove the existence of the rings in the parameter plane, we need to be more specific about the location of the rings in the dynamical plane. Let $V_{+}$be the portion of the prepole sector lying on and outside the critical circle and also between the two critical point rays through $c_{0}$ and $c_{1}$. That is,

$$
V_{+}=\left\{\left.z|| z|\geq| \lambda\right|^{1 / 2 n}, \frac{\operatorname{Arg} \lambda}{2 n} \leq \operatorname{Arg} z \leq \frac{\operatorname{Arg} \lambda}{2 n}+\frac{\pi}{n}\right\} .
$$

Let $V_{-}=\nu^{-1} \cdot V_{+}$. Therefore, $V_{-}$is the portion of the prepole sector bounded by the critical lines through $c_{0}$ and $c_{-1}$ and lying on or outside the critical circle. Let $V_{\lambda}=V_{+} \cup V_{-}$. See Figure 7

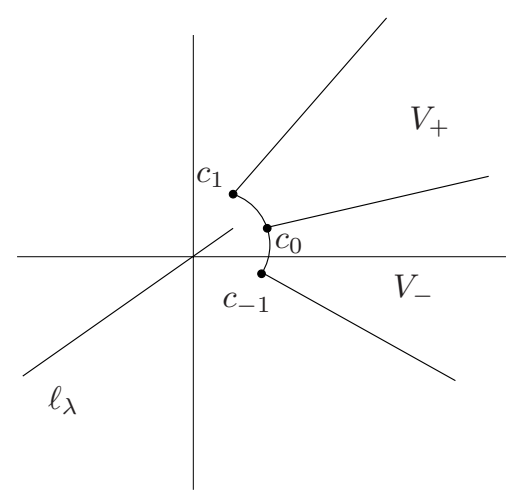

FiguRE 7. The region $V_{\lambda}=V_{+} \cup V_{-}$.

Since $|\operatorname{Arg} \lambda|<\pi$ and $n \geq 3$, we have for $z \in V_{\lambda}$

$$
|\operatorname{Arg} z| \leq\left|\frac{\operatorname{Arg} \lambda}{2 n}\right|+\frac{\pi}{n}<\frac{3 \pi}{2 n} \leq \frac{\pi}{2} .
$$

So for each $\lambda \in \mathcal{O}^{\prime}$, the region $V_{\lambda}$ is contained in the half plane $\operatorname{Re} z>0$.

Now $F_{\lambda}$ maps the portion of boundary of $V_{+}$lying along the critical circle oneto-one to the critical segment, since the endpoints of this arc are adjacent critical points along $C_{\lambda}$ that are mapped to distinct critical values. Also, $F_{\lambda}$ maps the portion of the critical point line containing $c_{0}$ lying on the boundary of $V_{+}$oneto-one onto the ray $t v_{\lambda}=2 t \sqrt{\lambda}$ with $t \geq 1$ and $\operatorname{Arg} \sqrt{\lambda}>0$, while $F_{\lambda}$ maps the other boundary ray containing $c_{1}$ to the negative of this ray. Hence the boundary of $V_{+}$is mapped onto the entire straight line passing through $\pm v_{\lambda}$ and the origin. Therefore $F_{\lambda}$ maps $V_{+}$univalently onto one of the half planes bounded by this line. Similarly, $F_{\lambda}$ maps $V_{-}$univalently onto the opposite half plane.

Let $\ell_{\lambda}$ be the straight line given by $2 t \sqrt{\lambda}$ where $t \in(-\infty, 1]$. So $\ell_{\lambda}$ is the straight line that starts at $2 \sqrt{\lambda}$ at $t=1$ and passes through the origin and $-2 \sqrt{\lambda}$ enroute to $\infty$ as $t \rightarrow \infty$. Note that the boundary of $V_{\lambda}$ is mapped two-to-one onto $\ell_{\lambda}$ by $F_{\lambda}$. Hence $F_{\lambda}$ maps the interior of $V_{\lambda}$ univalently onto $\mathbb{C}-\ell_{\lambda}$. Now, for each $\lambda \in \mathcal{O}^{\prime}$, 
the critical segment lies outside $V_{\lambda}$ since neither $V_{+}$nor $V_{-}$meets the interior of the critical circle. Also, the portion of $\ell_{\lambda}$ extending from $-2 \sqrt{\lambda}$ to $\infty$ lies in the left half plane, so the entire line $\ell_{\lambda}$ does not intersect $V_{\lambda}$. So we have:

Proposition. For each $\lambda \in \mathcal{O}^{\prime}, F_{\lambda}$ maps the interior of $V_{\lambda}$ univalently onto $\mathbb{C}-\ell_{\lambda}$, and so the image of $V_{\lambda}$ contains $V_{\lambda}$.

Recall that the $k^{\text {th }}$ ring in the dynamical plane is parametrized by $\gamma_{\lambda}^{k}(\theta)$ and is periodic with period $2 n^{k} \pi$.

Proposition. For each $k \geq 1$, the portion of the ring $\gamma_{\lambda}^{k}(\theta)$ with $|\theta| \leq n^{k-1} \pi$ lies in the region

$$
-\frac{3 \pi}{2 n}<\operatorname{Arg} z<\frac{3 \pi}{2 n}
$$

Proof. We first deal with the case $0 \leq \theta \leq n^{k-1} \pi$; the other case is handled by applying the $z \mapsto \nu^{-1} z$ symmetry, as we describe below.

We claim that the portion of the ring $\gamma_{\lambda}^{k}(\theta)$ with $0 \leq \theta \leq n^{k-1} \pi$ actually lies in the smaller region

$$
-\frac{\pi}{2 n}<\operatorname{Arg} z<\frac{3 \pi}{2 n}
$$

To see this, we first consider the simplest case where $\lambda \in \mathbb{R}^{+}$. In this case, $V_{+}$is bounded by $\mathbb{R}^{+}$and $\nu \cdot \mathbb{R}^{+}$, and $F_{\lambda}$ maps $V_{+}$univalently onto $\operatorname{Im} z \geq 0$. Recall that $\gamma_{\lambda}^{0}(\theta)$ lies in the region $\operatorname{Im} z \geq 0$ if $\theta \in[0, \pi]$. Hence there is a continuous preimage of $\gamma_{\lambda}^{0}(\theta)$ lying in $V_{+}$. This preimage is, by definition, $\gamma_{\lambda}^{1}(\theta)$ for $\theta \in[0, \pi]$. So $\gamma_{\lambda}^{1}(\theta)$ lies in the region $0 \leq \operatorname{Arg} z \leq \pi / n$, and thus the result is true when $k=1$.

Next note that $\gamma_{\lambda}^{1}(\pi)$ lies on the line $\nu \cdot \mathbb{R}^{+}$and is given by $\nu \gamma_{\lambda}^{1}(0)$. So we can use the symmetry in the dynamical plane to extend the definition of $\gamma_{\lambda}^{1}(\theta)$ to a continuous curve defined for $\theta \in[0, n \pi]$ as follows: if $\theta \in[j \pi,(j+1) \pi]$, let $\gamma_{\lambda}^{1}(\theta)=\nu^{j} \gamma_{\lambda}^{1}(\theta-j \pi)$ for $j=1, \ldots, n-1$. So $\gamma_{\lambda}^{1}(\theta)$ lies in $\operatorname{Im} z \geq 0$ for $\theta \in[0, n \pi]$. Then the sector $V_{+}$is again mapped over $\gamma_{\lambda}^{1}(\theta)$ for these $\theta$-values, so we have a continuous preimage $\gamma_{\lambda}^{2}(\theta)$ lying in $V_{+}$, mapped onto $\gamma_{\lambda}^{1}(\theta)$, and defined for $\theta \in[0, n \pi]$.

Then we extend the definition of $\gamma_{\lambda}^{2}(\theta)$ to $\left[0, n^{2} \pi\right]$ as above using the symmetry in the dynamical plane. So we have that $\gamma_{\lambda}^{3}(\theta)$ lies in $V_{+}$for all $\theta \in\left[0, n^{2} \pi\right]$. Continuing in this fashion proves the stronger result that $\gamma_{\lambda}^{k}(\theta)$ in fact lies in $V_{+}$ for $\theta \in\left[0, n^{k-1} \pi\right]$ for all $k$ as long as $\lambda \in \mathbb{R}^{+}$.

Now suppose that $0<\operatorname{Arg} \lambda<\pi$. We no longer have the fact that $V_{+}$is mapped over $\gamma_{\lambda}^{0}(\theta)$ for $0 \leq \theta \leq \pi$. Indeed, the point $\gamma_{\lambda}^{1}(0)$ now lies in $V_{-}$. This follows from the fact that the critical point ray through $c_{0}$ is mapped to a line whose argument is strictly larger than that of $c_{0}$, so the preimage of $c_{0}$ must lie below this critical point line. By the previous Proposition, we have that $F_{\lambda}$ maps the interior of the entire region $V_{\lambda}$ univalently onto $\mathbb{C}-\ell_{\lambda}$. Let $\ell_{\lambda}^{\prime}$ denote the portion of $\ell_{\lambda}$ lying in the lower half plane. Then

$$
\pi<\frac{\operatorname{Arg} \lambda}{2}+\pi=\operatorname{Arg} \ell_{\lambda}^{\prime}<\frac{3 \pi}{2} .
$$

Since, for $\theta \in[0, \pi]$, we have

$$
0<\operatorname{Arg} c_{0} \leq \operatorname{Arg} \gamma_{\lambda}^{0}(\theta) \leq \operatorname{Arg} c_{0}+\pi<\frac{\operatorname{Arg} \lambda}{2}+\pi=\operatorname{Arg} \ell_{\lambda}^{\prime},
$$


it follows that the entire line $\ell_{\lambda}$ never meets $\gamma_{\lambda}^{0}(\theta)$ for these $\theta$-values. Hence there is a continuous preimage of $\gamma_{\lambda}^{0}(\theta)$ in $V_{+} \cup V_{-}$for each $\theta \in[0, \pi]$. This defines $\gamma_{\lambda}^{1}(\theta)$ over this interval. Note that $\gamma_{\lambda}^{1}(\pi)=\nu \gamma_{\lambda}^{1}(0)$ must lie in $V_{+}$. In fact, we can say more:

$$
-\frac{\pi}{2 n}<\frac{\operatorname{Arg} \lambda}{2 n}-\frac{\pi}{2 n} \leq \operatorname{Arg} \gamma_{\lambda}^{1}(\theta)
$$

for $0 \leq \theta \leq \pi$. This follows since $F_{\lambda}$ maps the prepole line in $V_{-}$to a line perpendicular to $\ell_{\lambda}$ in $-\pi / 2<\operatorname{Arg} z<0$. This line does not intersect the curve $\gamma_{\lambda}^{0}(\theta)$ for $\theta \in[0, \pi]$. So $\gamma_{\lambda}^{1}(\theta)$ does not meet the prepole line in $V_{-}$. We therefore have

$$
-\frac{\pi}{2 n}<\operatorname{Arg} \gamma_{\lambda}^{1}(\theta)<\frac{3 \pi}{2 n}
$$

for $\theta \in[0, \pi]$, so this proves the case $k=1$ when $0<\operatorname{Arg} \lambda<\pi$.

We now extend the definition of $\gamma_{\lambda}^{1}(\theta)$ to $\theta \in[0, n \pi]$ as in the previous case using symmetry. Then we have, for $0 \leq \theta \leq n \pi$,

$$
-\frac{\pi}{2 n}<\operatorname{Arg} \gamma_{\lambda}^{1}(\theta) \leq \operatorname{Arg} c_{0}+\pi .
$$

But $\operatorname{Arg} c_{0}+\pi<\operatorname{Arg} \lambda / 2+\pi=\operatorname{Arg} \ell_{\lambda}^{\prime}$. So again $\ell_{\lambda}$ does not meet the extension of $\gamma_{\lambda}^{1}(\theta)$. So we have that $\gamma_{\lambda}^{2}(\theta)$ lies in the interior of $V_{+} \cup V_{-}$for $0 \leq \theta \leq n \pi$ and so $\operatorname{Arg} \gamma_{\lambda}^{2}(\theta)<3 \pi / 2 n$. As above we in fact also have $-\pi / 2 n \leq \operatorname{Arg} \gamma_{\lambda}^{2}(\theta)$, so this proves the case $k=2$. Continuing inductively proves the result for all $k$-values when $0<\operatorname{Arg} \lambda<\pi$ and $0 \leq \theta \leq n^{k-1} \pi$.

The case of negative values of $\theta$ is handled by symmetry as follows. We again assume that $0<\operatorname{Arg} \lambda<\pi$. For each $k$ we have, since $\gamma_{\lambda}^{k}(\theta)$ is $2 n^{k} \pi$ periodic,

$$
\begin{aligned}
F_{\lambda}\left(\nu^{-1} \gamma_{\lambda}^{k}(\theta)\right) & =-F_{\lambda}\left(\gamma_{\lambda}^{k}(\theta)\right) \\
& =-\gamma_{\lambda}^{k-1}(\theta) \\
& =\gamma_{\lambda}^{k-1}\left(\theta-n^{k-1} \pi\right) \\
& =F_{\lambda}\left(\gamma_{\lambda}^{k}\left(\theta-n^{k-1} \pi\right)\right) .
\end{aligned}
$$

Therefore

$$
\nu^{-1} \gamma_{\lambda}^{k}(\theta)=\gamma_{\lambda}^{k}\left(\theta-n^{k-1} \pi\right)
$$

follows since $\gamma_{\lambda}^{k}(\theta)$ is continuous in $\theta$. Therefore we have that, when $\theta \in\left[-n^{k-1} \pi, 0\right]$, $\gamma_{\lambda}^{k}(\theta)$ lies in the region

$$
-\frac{3 \pi}{2 n}<\operatorname{Arg} z<\frac{\pi}{2 n}
$$

So altogether the curve $\gamma_{\lambda}^{k}(\theta)$ lies in the region $|\operatorname{Arg} z|<3 \pi / 2 n$ for all $|\theta| \leq n^{k-1} \pi$. This concludes the proof when $0 \leq \operatorname{Arg} \lambda<\pi$.

If $-\pi<\operatorname{Arg} \lambda<0$, we invoke the $z \mapsto \bar{z}$ symmetry in the parameter plane. Since $F_{\lambda}$ is conjugate to $F_{\bar{\lambda}}$ via $z \mapsto \bar{z}$, it follows that the curves $\gamma_{\lambda}^{k}(\theta)$ are mapped to $\gamma_{\lambda}(-\theta)$ by the conjugacy. Hence these curves lie in the same region when $-\pi<$ $\operatorname{Arg} \lambda<0$. This concludes the proof.

\section{Rings in the parameter plane}

Before turning to the proof of the existence of rings in the parameter plane, we need to examine more carefully the parametrizations of the rings in the dynamical plane in two of the special cases discussed earlier, namely when $\lambda \in \mathbb{R}^{+}$and $\lambda \in$ $\omega \cdot \mathbb{R}^{+}$. 
First suppose that $\lambda \in \mathbb{R}^{+}$. For the special parameters $\lambda_{k}$ among the superstable parameters in $\mathbb{R}^{+}$, we have seen that $F_{\lambda_{k}}\left(c_{0}\right)$ always lies in $\mathbb{R}^{+}$and satisfies

$$
0<F_{\lambda_{k}}\left(c_{0}\right)<c_{0}=F_{\lambda_{k}}^{k}\left(c_{0}\right)<F_{\lambda_{k}}^{k-1}\left(c_{0}\right)<\ldots<F_{\lambda_{k}}^{2}\left(c_{0}\right) .
$$

Hence $F_{\lambda_{k}}^{2}\left(c_{0}\right)$ lies on $\gamma_{\lambda_{k}}^{k-2} \cap \mathbb{R}^{+}$and $F_{\lambda_{k}}^{j}\left(c_{0}\right)$ lies on $\gamma_{\lambda_{k}}^{k-j} \cap \mathbb{R}^{+}$for $j=2, \ldots, k$.

In particular, since the definition of the parametrization requires that $F_{\lambda}\left(\gamma_{\lambda}^{j}(0)\right)$ $=\gamma_{\lambda}^{j-1}(0)$, it follows that, for the special parameter value $\lambda_{k}$, we have

$$
\begin{aligned}
\gamma_{\lambda_{k}}^{0}(0) & =c_{0}, \\
\gamma_{\lambda_{k}}^{k-2}(0) & =F_{\lambda_{k}}^{2}\left(c_{0}\right), \\
\gamma_{\lambda_{k}}^{k-3}(0) & =F_{\lambda_{k}}^{3}\left(c_{0}\right), \\
& \vdots \\
\gamma_{\lambda_{k}}^{1}(0) & =F_{\lambda_{k}}^{k-1}\left(c_{0}\right) .
\end{aligned}
$$

Next we turn our attention to the special parameter values $\lambda_{k} \omega$ lying along the line $\omega \cdot \mathbb{R}^{+}$in the parameter plane. Here the situation is somewhat more complicated. For simplicity of notation, we fix a value of $k$ and set $\mu=\lambda_{k} \omega$.

As we showed earlier, the line $\omega^{n / 2} \cdot \mathbb{R}^{+}$contains the critical point $c_{n+1}$ and is either invariant under $F_{\mu}$ (if $n$ is even) or interchanged with the symmetric line $-\omega^{n / 2} \cdot \mathbb{R}^{+}$by $F_{\mu}$ (if $n$ is odd). In either case the symmetric line $-\omega^{n / 2} \cdot \mathbb{R}^{+}$is mapped to this line by $F_{\mu}$ and contains the critical point $c_{1}=-c_{n+1}$. Also, the critical point line through $c_{0}$ is mapped to $-\omega^{n / 2} \cdot \mathbb{R}^{+}$by $F_{\mu}$ and then to $\omega^{n / 2} \cdot \mathbb{R}^{+}$ by $F_{\mu}^{2}$.

We have, by definition, $\gamma_{\mu}^{0}(0)=c_{0}$. Since $c_{1}=\nu c_{0}$ where, as usual, $\nu=$ $\exp (\pi i / n)$, we also have

$$
\begin{aligned}
c_{1} & =\gamma_{\mu}^{0}\left(\frac{\pi}{n}\right), \\
c_{n+1} & =\gamma_{\mu}^{0}\left(\frac{\pi}{n}+\pi\right) .
\end{aligned}
$$

Consider the portion of the critical point sector containing $c_{0}$ and lying on or outside $C_{\lambda} \cdot \gamma_{\mu}^{1}(0)$ is the unique point in this region that is mapped to $c_{0}$ by $F_{\mu}$. Since $F_{\mu}$ takes the critical point line through $c_{0}$ to the critical point line through $c_{1}$, it follows that $\gamma_{\mu}^{1}(0)$ lies below this line and that $\gamma_{\mu}^{1}(\pi / n)$, the preimage of $c_{1}$, lies on the critical point line through $c_{0}$. By symmetry, $\gamma_{\mu}^{1}((\pi / n)+\pi)$ then lies on the critical point line through $c_{1}$, and, since $\gamma_{\mu}^{1}$ is $2 n \pi$-periodic, the point

$$
\gamma_{\mu}^{1}\left(\frac{\pi}{n}+\pi+n \pi\right)
$$

lies on the line $\omega^{n / 2} \cdot \mathbb{R}^{+}$containing $c_{n+1}$.

Continuing, we have that $\gamma_{\mu}^{2}((\pi / n)+\pi)$ lies on the critical point line through $c_{0}$ and is mapped by $F_{\mu}$ to $\gamma_{\mu}^{1}((\pi / n)+\pi)$. The point

$$
\gamma_{\mu}^{2}\left(\frac{\pi}{n}+\pi+n \pi\right)
$$

then lies on the critical point line through $c_{1}$ and is mapped to

$$
\gamma_{\mu}^{1}\left(\frac{\pi}{n}+\pi+n \pi\right)
$$

on $\omega^{n / 2} \cdot \mathbb{R}^{+}$. 
Continuing inductively, we see that the critical point line through $c_{0}$ contains the points

$$
\begin{aligned}
& c_{0}=\gamma_{\mu}^{0}(0), \\
& \gamma_{\mu}^{1}\left(\frac{\pi}{n}\right), \\
& \gamma_{\mu}^{2}\left(\frac{\pi}{n}+\pi\right), \\
& \quad \vdots \\
& \gamma_{\mu}^{j}\left(\frac{\pi}{n}+\pi+n \pi+\ldots+n^{j-2} \pi\right)=\gamma_{\mu}^{j}\left(\frac{\pi}{n}\left(1+n+\ldots+n^{j-1}\right)\right),
\end{aligned}
$$

and the critical point line through $c_{1}$ contains the points

$$
\begin{aligned}
& c_{1}=\gamma_{\mu}^{0}\left(\frac{\pi}{n}\right), \\
& \gamma_{\mu}^{1}\left(\frac{\pi}{n}+\pi\right), \\
& \gamma_{\mu}^{2}\left(\frac{\pi}{n}+\pi+n \pi\right), \\
& \quad \vdots \\
& \gamma_{\mu}^{j}\left(\frac{\pi}{n}+\pi+n \pi+\ldots+n^{j-1} \pi\right)=\gamma_{\mu}^{j}\left(\frac{\pi}{n}\left(1+n+\ldots+n^{j}\right)\right) .
\end{aligned}
$$

Equivalently, $\gamma_{\mu}^{j}(\theta)$ lies on the critical point line through $c_{1}$ for

$$
\theta=\frac{\pi}{n}\left(\frac{n^{j+1}-1}{n-1}\right)
$$

Now consider the corresponding points on the critical point line through $c_{-1}$. Since the parametrization corresponding to points on this line and since $\gamma_{\mu}^{j}$ is obtained by subtracting $n^{j-1} \pi$ from the corresponding critical point line through $c_{0}$, we find the following points on this critical point line:

$$
\begin{aligned}
& c_{-1}=\gamma_{\mu}^{0}\left(-\frac{\pi}{n}\right), \\
& \gamma_{\mu}^{1}\left(\frac{\pi}{n}-\pi\right), \\
& \gamma_{\mu}^{2}\left(\frac{\pi}{n}+\pi-n \pi\right), \\
& \quad \vdots \\
& \gamma_{\mu}^{j}\left(\frac{\pi}{n}+\pi+n \pi+\ldots+n^{j-2} \pi-n^{j-1} \pi\right) .
\end{aligned}
$$

Equivalently, $\gamma_{\mu}^{j}(\theta)$ lies on the critical point line through $c_{-1}$ for

$$
\theta=\frac{\pi}{n}\left(1+n+n^{2}+\ldots+n^{j-1}-n^{j}\right)=\frac{\pi}{n}\left(\frac{n^{j}-1}{n-1}\right)-n^{j-1} \pi .
$$

For later use, this value of $\theta$ is called $\theta_{n, j}$. See Figure 8 ,

We now turn to the proof of the existence of the rings $\mathcal{S}^{k}$ in the parameter plane for $k>1$. For technical reasons, we consider only the case when $n \geq 5$ in this section; the special cases $n=3,4$ are handled later. 


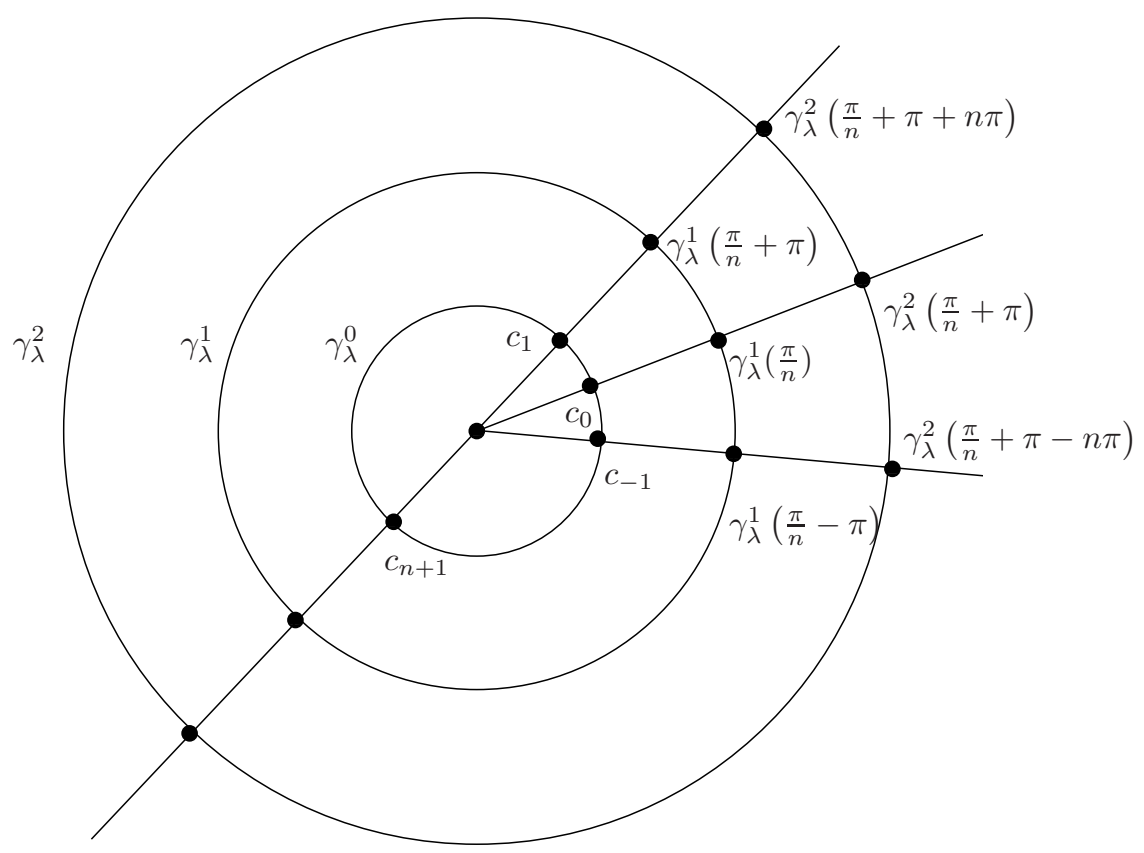

FIGURE 8. Parametrization of $\gamma_{\lambda}(\theta)$ when $\lambda=\lambda_{k} \omega$.

Recall that, from the results of the previous section, we have that, when $k \geq 1$, the portion of the curve $\gamma_{\lambda}^{k}(\theta)$ for $|\theta| \leq n^{k-1} \pi$ lies in the region

$$
-\frac{3 \pi}{2 n}<\operatorname{Arg} z<\frac{3 \pi}{2 n}
$$

We call this region $W_{n}$ and note that $W_{n}$ lies in the right half plane. Let $H_{\lambda}$ denote the involution that fixes $c_{0}$, i.e.,

$$
H_{\lambda}(z)=\frac{\lambda^{1 / n}}{z} .
$$

Lemma. If $n \geq 5$ and $\lambda \in \mathcal{O}^{\prime}$, then $H_{\lambda}\left(W_{n}\right)$ lies in the half plane $\operatorname{Re} z>0$.

Proof. Since

$$
\operatorname{Arg} H_{\lambda}(z)=\frac{\operatorname{Arg} \lambda}{n}-\operatorname{Arg} z
$$

we have, if $z \in W_{n}$ and $n \geq 5$,

$$
-\frac{\pi}{2} \leq-\frac{5 \pi}{2 n} \leq-\frac{3 \pi}{2 n}+\frac{\operatorname{Arg} \lambda}{n}<\operatorname{Arg} H_{\lambda}(z)<\frac{3 \pi}{2 n}+\frac{\operatorname{Arg} \lambda}{n} \leq \frac{5 \pi}{2 n} \leq \frac{\pi}{2} .
$$

We remark that this result is false when $n=3,4$; that is the reason why these are special cases.

Now consider the curves

$$
\xi_{\lambda}^{k}(\theta)=H_{\lambda}\left(\gamma_{\lambda}^{k}(\theta)\right)
$$


Since the involution $H_{\lambda}$ interchanges the inside and outside of $C_{\lambda}$, each of the curves $\xi_{\lambda}^{k}$ is a simple closed curve lying inside the critical circle. We have

$$
F_{\lambda}\left(\xi_{\lambda}^{k}(\theta)\right)=\gamma_{\lambda}^{k-1}(\theta)
$$

since $F_{\lambda}\left(H_{\lambda}(z)\right)=F_{\lambda}(z)$. By the Lemma, we also have that $\xi_{\lambda}^{k}(\theta)$ lies in $\operatorname{Re} z>0$ for $|\theta| \leq n^{k-1} \pi$, at least if $n \geq 5$.

Theorem. For each $k \geq 1$ and any $\theta$ satisfying $|\theta| \leq n^{k-1} \pi$, there exists a unique parameter $\lambda=\lambda_{\theta, k}$ such that

$$
v_{\lambda}=2 \sqrt{\lambda}=\xi_{\lambda}^{k}(\theta)
$$

Proof. The function $G(\lambda)=v_{\lambda}=2 \sqrt{\lambda}$ takes the subset $\mathcal{O}^{\prime}$ of the parameter plane univalently onto an open subset of $\operatorname{Re} z>0$. For each $\lambda \in \mathcal{O}^{\prime}, G(\lambda)$ lies inside $C_{\lambda}$, but for $\lambda$ on the dividing circle (which is the circular boundary of $\mathcal{O}^{\prime}$ ), $G(\lambda)$ lies on the critical circle. Hence $G$ maps $\mathcal{O}^{\prime}$ univalently onto the interior of a half disk in the right half plane that contains the region inside $C_{\lambda}$ in $\operatorname{Re} z>0$ for each $\lambda \in \mathcal{O}^{\prime}$. Call this half disk $D$.

Also, for fixed $\theta$, the function $\lambda \mapsto \xi_{\lambda}^{k}(\theta)$ is analytic on $\mathcal{O}^{\prime}$ and takes this set strictly inside the portion of the critical circle bounded by the rays $|\operatorname{Arg} z|=3 \pi / 2 n$. Hence, for each $\theta$, the set of points $\xi_{\lambda}^{k}(\theta)$ lies inside a compact sector in $D$. That is, this set of points can possibly accumulate on the boundary of $D$ only at the origin. Hence we may consider the composition $Q(\lambda)=G^{-1}\left(\xi_{\lambda}^{k}(\theta)\right)$. As a function of $\lambda, Q$ is analytic and maps the simply connected region $\mathcal{O}^{\prime}$ inside itself. By the Schwarz Lemma, $Q$ has a unique fixed point in this set or on its boundary. But the fixed point cannot lie at $\lambda=0$ since 0 is surrounded by the McMullen domain so that the curves $\xi_{\lambda}^{k}$ are bounded away from the origin. Hence there must be a unique fixed point in the interior of $D$. This fixed point is $\lambda_{\theta, k}$.

Note that the fixed points $\lambda_{\theta, k}$ vary continuously with $\theta$, so $\theta \mapsto \lambda_{\theta, k}$ is a curve in the parameter plane.

The following Proposition identifies the specific values of $\lambda_{\theta, k}$ corresponding to the special cases considered earlier.

Proposition. When $\theta=0$ and $k \geq 1$, the parameter values $\lambda_{0, k}$ are given by the parameters $\lambda_{k+1} \in \mathbb{R}^{+}$. When $\theta=\theta_{n, k}, \lambda(\theta, k)$ is given by $\omega \lambda_{k+1}$ on the symmetry line $\omega \cdot \mathbb{R}^{+}$.

Proof. When $\lambda \in \mathbb{R}^{+}$, the points $\gamma_{\lambda}^{j}(0)$ also lie in $\mathbb{R}^{+}$for each $j$. Since, as shown earlier, the parameter $\lambda_{k+1}$ has the property that $v_{\lambda_{k+1}} \in \xi_{\lambda_{k+1}}^{k}, F_{\lambda_{k+1}}^{2}\left(c_{0}\right) \in \gamma_{\lambda_{k+1}}^{k-1} \cap$ $\mathbb{R}^{+}$and the forward orbit of this point decreases along $\mathbb{R}^{+}$until meeting $c_{0}$, it follows from the uniqueness of the parameter $\lambda_{0, j}$ that we must have $\lambda_{0, k}=\lambda_{k+1}$ for each $k \geq 1$.

When $\lambda=\lambda_{k+1} \omega$ and $\theta=\theta_{n, k}$, we know that the point $\gamma_{\lambda}^{k}\left(\theta_{n, k}\right)$ lies on the critical point line through $c_{-1}$. Hence $H_{\lambda}\left(\gamma_{\lambda}^{k}\left(\theta_{n, k}\right)\right)$ lies on the critical point line through $c_{1}$ and is given by $\xi_{\lambda}^{k}\left(\theta_{n, k}\right)$. This point is then mapped by $F_{\lambda}$ to the point on $\omega^{n / 2} \cdot \mathbb{R}^{+}$whose orbit meets $c_{n+1}$ after $k-1$ iterations of $F_{\lambda}$ or $F_{\lambda}^{2}$, depending upon whether $n$ is even or odd. Hence $\lambda_{\theta_{n, k}, k}=\lambda_{k+1} \omega$ as claimed.

Now the parameters in the previous Proposition are the unique parameters on the corresponding lines in the parameter space for which the orbit of the second iterate of the appropriate critical point monotonically decreases along the corresponding 
line(s) for $k-1$ iterations before returning to itself and becoming periodic. So the curve $\theta \mapsto \lambda_{\theta, k}$ meets each of these two symmetry lines only once. Hence the portion of this curve defined for $0 \leq \theta \leq \theta_{n, k}$ either lies outside the sector

$$
0 \leq \operatorname{Arg} \lambda \leq \frac{2 \pi}{n-1}
$$

for all values of $\theta$ or else this entire curve lies inside the sector. But the former cannot occur since this would imply that some $\lambda_{\theta, k}$ would lie in $\mathbb{R}^{-}$, contradicting the fact that each $\lambda_{\theta, k}$ lies in $\mathcal{O}^{\prime}$. Hence the portion of the curve $\lambda_{\theta, k}$ defined for $0 \leq \theta \leq \theta_{n, k}$ is a continuous arc connecting $\theta=0$ and $\theta=2 \pi /(n-1)$. It then follows by the $(n-1)$-fold symmetry that, for each $k \geq 1, \lambda_{\theta, k}$ is a simple closed curve in parameter space which is periodic of period

$$
\begin{aligned}
(n-1) \theta_{n, k} & =(n-1)\left(\frac{\pi}{n}\left(\frac{n^{k}-1}{n-1}\right)-n^{k-1} \pi\right) \\
& =\frac{\pi}{n}\left(-n^{k+1}+2 n^{k}-1\right) .
\end{aligned}
$$

We therefore define the ring $\mathcal{S}^{k+1}$ to be the simple closed curve $\theta \mapsto \lambda_{\theta, k}$. That is, $\mathcal{S}^{k+1}$ consists of parameter values for which the critical orbit has the following behavior:

1. both critical values lie inside the critical circle;

2. $F_{\lambda}^{2}\left(c_{\lambda}\right)$ lies on $\gamma_{\lambda}^{k-1}$;

3. subsequent iterates decrease through the $\gamma_{\lambda}^{j}$ until, at the $k^{\text {th }}$ iterate, the critical orbit lands back on the critical circle.

We have shown:

Theorem. When $n \geq 5$, the ring $\mathcal{S}^{k+1}$ in the parameter space is a simple closed curve that is parameterized by $\theta \mapsto \lambda_{\theta, k}$ and is periodic of period

$$
\frac{\pi}{n}\left(n^{k+1}-2 n^{k}+1\right)=\frac{\pi}{n}\left((n-2) n^{k}+1\right) .
$$

In particular, since the critical points (resp., prepoles) of $F_{\lambda}$ are located on $\gamma_{\lambda}^{0}(\theta)$ at $\theta=\pi j / n$ (resp., $(2 j+1) \pi / 2 n$ ) for $0 \leq j<2 n$, we have the following count of superstable parameters and centers of Sierpinski holes along $\mathcal{S}^{k+1}$ :

Corollary. There are precisely $(n-2) n^{k}+1$ parameters along $\mathcal{S}^{k+1}$ that are superstable parameters. There are the same number of parameters that are centers of Sierpinski holes. These parameters alternate between these two types as the parameter winds around $\mathcal{S}^{k+1}$.

\section{The SPECIAL CASES $n=3$ AND $n=4$}

In this section we turn our attention to the special cases of the Rings Around the McMullen Domain Theorem that cannot be handled by our general methods; these are the cases when $n=3$ and $n=4$. The problems that arose in these cases occurred because the function $G(\lambda)=F_{\lambda}\left(c_{\lambda}\right)=2 \sqrt{\lambda}$ did not cover enough of the curves $\gamma_{\lambda}^{k}$ to provide us with the means to find the fixed point $\lambda_{\theta}$. We remedy this in these cases by using the function $G(\lambda)=F_{\lambda}^{2}\left(c_{\lambda}\right)$ instead. 
We first deal with the case $n=4$. That is, suppose

$$
F_{\lambda}(z)=z^{4}+\frac{\lambda}{z^{4}}
$$

so $F_{\lambda}$ has eight free critical points, two critical values, and eight prepoles.

Let $\lambda^{*}=\lambda_{4}^{*}$ be the radius of the dividing circle in the parameter plane. Since $\lambda^{*}$ is the unique solution of the equation $2|\sqrt{\lambda}|=|\lambda|^{1 / 8}$, we compute that $\lambda^{*}=\left|2^{-8 / 3}\right|$. Let $\mathcal{O}$ be the open disk in the parameter plane inside the dividing circle, so that $|\lambda|<\lambda^{*}$ in $\mathcal{O}$. As before, if $\lambda \in \mathcal{O}$, then the critical circle is mapped strictly inside itself by $F_{\lambda}$.

The following result gives a bound on the location of the boundary of $B_{\lambda}$ that will prove useful later.

Proposition (Location of $\left.\partial B_{\lambda}\right)$. Suppose $|\lambda| \leq \lambda^{*}$. Then the boundary of $B_{\lambda}$ is contained in the open annulus bounded by the circles of radius 0.9 and 1.2 centered at the origin. The inner (resp., outer) boundary of this annulus is mapped strictly inside (resp., outside) itself by $F_{\lambda}$. Moreover, if $|z| \geq 1.2$, then $z \in B_{\lambda}$.

Proof. A straightforward computation shows that if $|z|=0.9$, then

$$
\begin{aligned}
\left|F_{\lambda}(z)\right| & \leq(0.9)^{4}+\frac{|\lambda|}{(0.9)^{4}} \\
& \leq(0.9)^{4}+\left(\frac{1}{2}\right)^{8 / 3}(0.9)^{-4} \\
& <0.897,
\end{aligned}
$$

so $F_{\lambda}$ maps the circle of radius 0.9 inside a smaller circle about the origin. Hence $B_{\lambda}$ lies strictly outside the circle of radius 0.9 .

On the other hand, if $|z| \geq 1.2$ and $|\lambda| \leq \lambda^{*}<1 / 4$, we have

$$
\left|F_{\lambda}(z)\right| \geq|z|^{4}-\frac{|\lambda|}{|z|^{4}} \geq|z|^{4}-\frac{1}{4(1.2)^{4}}>|z|^{4}-0.13>|z|^{2}>|z| .
$$

It follows that $F_{\lambda}$ maps each circle of the form $|z|=r \geq 1.2$ strictly outside itself and hence the entire region $|z| \geq 1.2$ is contained in $B_{\lambda}$. Therefore the boundary of $B_{\lambda}$ must lie somewhere strictly inside the annulus bounded by the circles of radii 0.9 and 1.2 .

It is known that the McMullen domain consists of a single, open, simply connected region surrounding the origin in the parameter plane 3 . We denote this domain by $\mathcal{M}$. Recall that $F_{\lambda}^{2}$ maps all eight critical points onto the same point. The location of this second image of the critical points as $\lambda$ varies can also be used to parametrize the rings $\mathcal{S}^{k}$ around $\mathcal{M}$ in the parameter plane. Therefore we let $G$ be the function that gives the location of this point, that is,

$$
G(\lambda)=F_{\lambda}^{2}\left(\lambda^{1 / 8}\right)=16 \lambda^{2}+\frac{1}{16 \lambda} .
$$

Note that $G$ is defined in the parameter plane, depends analytically on $\lambda$, and has a simple pole when $\lambda=0$. So even though $F_{\lambda}$ has no free critical points when $\lambda=0$, the map $G$ is still well defined and analytic at this parameter value.

The following result gives an estimate of the size and location of $\mathcal{M}$ in the parameter space. 
Proposition (Location of $\mathcal{M})$. The McMullen domain $\mathcal{M}$ is contained in the open disk of radius $1 / 8$ centered at the origin in the parameter space. The closed disk of radius $1 / 20$ centered at the origin lies inside $\mathcal{M}$.

Proof. First suppose that $|\lambda|=1 / 8$. Then we have

$$
|G(\lambda)| \leq 16|\lambda|^{2}+\frac{1}{16|\lambda|}=\frac{3}{4}<0.9 .
$$

Since $1 / 8<(1 / 2)^{8 / 3}=\lambda^{*}$, it follows from the previous Proposition that the second image of the critical points does not lie in $B_{\lambda}$ when $|\lambda|=1 / 8$. Therefore $\mathcal{M}$ lies inside this circle.

On the other hand, if $|\lambda| \leq 1 / 20$, we have that

$$
\begin{aligned}
|G(\lambda)| & =\left|\frac{1}{16 \lambda}+16 \lambda^{2}\right| \\
& \geq \frac{1}{16} \frac{1}{|\lambda|}-16|\lambda|^{2} \\
& \geq \frac{20}{16}-\frac{16}{400}=1.21
\end{aligned}
$$

By the previous Proposition, $G(\lambda)$ lies in $B_{\lambda}$, but since $|\lambda|<\lambda^{*}$, the critical values themselves do not lie in $B_{\lambda}$. Therefore the critical values lie in $T_{\lambda}$ and so $\lambda \in \mathcal{M}$.

Let $\mathcal{W}$ be the interior of the disk of radius $1 / 8$ in the parameter plane. Then we have $\mathcal{M} \subset \mathcal{W} \subset \mathcal{O}$.

Proposition. The function $G$ is one-to-one on $\mathcal{W}$.

Proof. Suppose otherwise. Then there exist $\lambda, \mu \in \mathcal{W}$ with $\lambda \neq \mu$ but

$$
16 \lambda^{2}+\frac{1}{16 \lambda}=16 \mu^{2}+\frac{1}{16 \mu}
$$

so that

$$
16^{2}\left(\lambda^{2}-\mu^{2}\right)=\frac{\lambda-\mu}{\lambda \mu} .
$$

Since $\lambda \neq \mu$, we must then have

$$
16^{2}|\lambda+\mu|=\frac{1}{|\lambda \mu|}
$$

But $16^{2}|\lambda+\mu|<64$, whereas $1 /|\lambda \mu|>64$. This yields a contradiction.

Indeed, a computation shows that the function $G$ has three critical points and each lies on the circle of radius $1 / 8$ centered at the origin. Therefore $\mathcal{W}$ is the largest open circular disk surrounding the origin on which $G$ is univalent.

Now let $\mathcal{W}^{\prime}$ denote the open simply connected region obtained by removing both the closed disk of radius $1 / 20$ about $\lambda=0$ and the negative real axis from $\mathcal{W}$. We have:

Proposition. The function $G$ maps $\mathcal{W}^{\prime}$ univalently over a region that contains $\{z|3 / 4<| z \mid<1.21\}-\mathbb{R}^{-}$. 
Proof. As in the proof of the Location of $\mathcal{M}$ Proposition, if $|\lambda|=1 / 8$, then $|G(\lambda)| \leq$ $3 / 4$. On the other hand, if $|\lambda|=1 / 20$, then we have

$$
|G(\lambda)| \geq \frac{1}{16|\lambda|}-16|\lambda|^{2} \geq 1.21
$$

Since $G$ is one-to-one on all of $\mathcal{W}$ and maps the interval $[-1 / 8,-1 / 20]$ into $\mathbb{R}^{-}$, the result follows.

Proposition. For each $\lambda \in \mathcal{W}^{\prime}$ and each $k \geq 1$, the curves $\gamma_{\lambda}^{k}(\theta)$ all lie in the annulus $3 / 4<|z|<1.21$.

Proof. As we showed earlier, the circle of radius 1.21 lies in $B_{\lambda}$ for each $\lambda \in \mathcal{W}^{\prime}$, so all of the $\gamma_{\lambda}^{k}$ lie inside this circle. On the other hand, if $|z|=3 / 4$, then

$$
\left|F_{\lambda}(z)\right| \leq\left(\frac{3}{4}\right)^{4}+|\lambda|\left(\frac{4}{3}\right)^{4}<|\lambda|^{1 / 8} .
$$

The second inequality follows by comparing the graphs of the linear expression on the left to that of $|\lambda|^{1 / 8}$ over the interval $1 / 20 \leq|\lambda| \leq 1 / 8$. Therefore, for each $\lambda \in \mathcal{W}^{\prime}, F_{\lambda}$ maps the circle of radius $3 / 4$ centered at the origin strictly inside $C_{\lambda}$. Hence all of the curves $\gamma_{\lambda}^{k}$ with $k \geq 1$ must lie outside this circle.

The proof of the existence of the rings $\mathcal{S}^{k}$ now follows exactly as in the previous section, where we now use the function $G$ defined on $\mathcal{W}^{\prime}$. The image of $G$ covers the region $|\operatorname{Arg} z| \leq 3 \pi / 8$ in the annulus of the previous Proposition, and we showed in Section 3 that this region contains $\gamma_{\lambda}^{k}(\theta)$ for each $k \geq 1$. Thus we find the fixed point $\lambda_{\theta}$ that parameterizes $\mathcal{S}^{k}$ for each $k \geq 3$. The case $k=1$ is the dividing circle case described in Section 2. The case $k=2$ in which the critical points land back on $C_{\lambda}$ after two iterations (and hence do not meet any of the $\gamma_{\lambda}^{k}$ with $k \geq 1$ ) is a similar straightforward computation.

When $n=3$, the proof is essentially the same. The main difference in this case is that

$$
G(\lambda)=8 \lambda^{3 / 2}+\frac{1}{8 \sqrt{\lambda}}
$$

no longer maps the disk about the origin univalently outside a circle in $\mathbb{C}$. Rather, in this case, $G$ maps such a disk minus $\mathbb{R}^{-}$univalently onto the exterior of a circle in the right half plane. However, this is precisely where the curves $\gamma_{\lambda}^{k}$ are constrained to lie when $n=3$, so the proof goes through as above. We leave the details in this case to the reader.

\section{REFERENCES}

[1] Blanchard, P., Devaney, R. L., Look, D. M., Seal, P., and Shapiro, Y., Sierpinski-Curve Julia Sets and Singular Perturbations of Complex Polynomials, Ergodic Theory and Dynamical Systems 25 (2005), 1047-1055. MR2158396 (2006d:37087)

[2] Devaney, R. L., Baby Mandelbrot Sets Adorned with Halos in Families of Rational Maps, Complex Dynamics: Twenty Five Years After the Appearance of the Mandelbrot Set, Contemp. Math., Vol. 396, Amer. Math. Soc. Providence, RI, 2006. MR2209085

[3] Devaney, R. L., Structure of the McMullen Domain in the Parameter Space of Rational Maps, Fund. Math. 185 (2005), 267-285. MR2161407(2006c:37046)

[4] Devaney, R. L., The McMullen Domain: Satellite Mandelbrot Sets and Sierpinski Holes. To appear.

[5] Devaney, R. L. and Look, D. M., A Criterion for Sierpinski Curve Julia Sets. To appear in Topology Proceedings. 
[6] Devaney, R. L., Look, D. M., and Uminsky, D., The Escape Trichotomy for Singularly Perturbed Rational Maps. Indiana Univ. Math. J. 54 (2005), 1621-1634. MR2189680

[7] Douady, A. and Hubbard, J., Etude Dynamique des Polynômes Complexes. Publ. Math. D'Orsay (1984). MR0762431 (87f:58072a)

[8] Douady, A. and Hubbard, J., On the Dynamics of Polynomial-like Mappings. Ann. Sci. ENS Paris 18 (1985), 287-343. MR0816367 (87f:58083)

[9] McMullen, C., Automorphisms of Rational Maps. Holomorphic Functions and Moduli. Vol. 1. Math. Sci. Res. Inst. Publ. 10. Springer, New York, 1988. MR0955807 (89m:58187)

[10] McMullen, C., The Classification of Conformal Dynamical Systems. Current Developments in Mathematics. Internat. Press, Cambridge, MA (1995), 323-360. MR1474980(98h:58162)

[11] Milnor, J., Dynamics in One Complex Variable. Vieweg, 1999. MR1721240(2002i:37057)

[12] Milnor, J. and Tan Lei, A "Sierpinski Carpet" as Julia Set. Appendix F in Geometry and Dynamics of Quadratic Rational Maps. Experiment. Math. 2 (1993), 37-83. MR1246482 (96b:58094)

[13] Petersen, C. and Ryd, G., Convergence of Rational Rays in Parameter Spaces. In The Mandelbrot set: Theme and Variations, London Mathematical Society, Lecture Note Series 274, Cambridge University Press, 161-172, 2000. MR 1765088 (2001f:37057)

[14] Roesch, P., On Captures for the Family $f_{\lambda}(z)=z^{2}+\lambda / z^{2}$. To appear.

[15] Whyburn, G. T., Topological Characterization of the Sierpinski Curve. Fund. Math. 45 (1958), 320-324. MR0099638 (20:6077)

Department of Mathematics, Boston University, 111 Cummington Street, Boston, MASSACHUSETtS 02215

Department of Mathematics, Boston University, 111 Cummington Street, Boston, Massachusetts 02215 